\title{
Hidroelektrik Santrallerde Türbin Seçimi ve Francis Türbin Tasarım Parametreleri
}

\author{
Hakan ADATEPE ${ }^{1 *}$, Volkan ÖZ $^{1}$ \\ ${ }^{1}$ Giresun Üniversitesi, Mühendislik Fakültesi, Enerji Sistemleri Mühendisliği Bölümü, Giresun, TÜRKIYE
}

"Sorumlu Yazar: adatepehakan@yahoo.com

Geliş Tarihi: 01.11.2019

Kabul Tarihi: 09.12.2019

\begin{abstract}
$\ddot{O} \mathbf{z}$
Teknolojinin gelişmesiyle enerjiye olan ihtiyaçta aynı paralelde artmıştır ve artmaya devam etmektedir. Her ne kadar yıllar geçtikçe oranı azalsa da hala \% 50 oranında dışa bağımlılı̆̆ımız sürmektedir. Dışa bağımlılığın azalmasında yenilenebilir enerji kaynaklarının üretimdeki payının artması gerekmektedir. Yenilenebilir enerji kaynaklarından da Hidrolik enerji, yaklaşık olarak \%68 ile yenilenebilir enerji kaynakları arasında elektrik üretimi sağlama bakımından ilk sıradadır. Hidrolik enerji, hem düşük enerji üretim maliyeti hem de hidrolik potansiyelin fazla olması sebebiyle ülkemizde ilk tercih edilen enerji üretim yöntemidir. Ülkemizdeki hidroelektrik santrallerinin tamamına yakını yabancı üretim olmakla beraber son yıllarda yerli üretim sanayinde çalışmalar yapılmaya başlanmış ancak henüz yeterli seviyeye ulaşamamıştır. Bu çalışmada, Hidrolik santrallerde yaygın olarak kullanılan türbin tipi olan Francis türbin çarkı tasarımında Siervo ve leva metodunun ampirik ifadeleri vurgulanmıştır.
\end{abstract}

Anahtar Kelimeler: Francis Türbin Çarkı, Hidroelektrik Santral, Özgül Hız.

\section{Turbine Selection in Hydroelectric Power Plants and Francis Turbine Design Parameters}

\begin{abstract}
With the development of technology, the need for energy has increased in parallel and continues to increase. Although the rate decreases over the years, we still have $50 \%$ dependence on foreign sources. The share of renewable energy sources in production should increase in order to decrease dependence on foreign sources. Among renewable energy sources, Hydraulic energy is at the forefront in terms of generating electricity between renewable energy sources with approximately $68 \%$. Hydraulic energy is the first preferred energy production method in our country due to both low energy production cost and high hydraulic potential. Although almost all of the hydroelectric power plants in our country are foreign production, studies have started to be carried out in the domestic production industry in recent years, but they have not reached a sufficient level yet. In this study, empirical expressions of Siervo and leva method are emphasized in Francis turbine wheel design which is widely used in hydraulic power plants.
\end{abstract}

Keywords: Francis Turbine Runner, Hydroelectric Power Plant, Specific Speed. 


\section{Giriş}

Ülkelerin gelişmişlik seviyesinin, o ülkenin kişi başına düşen elektrik enerjisi tüketimi ile belirlendiği ve elektrik enerjisi tüketiminin artmasıyla, o ülkenin ekonomik olarak kalkındığını ve refah seviyesinin yükseldiğini gösteren araştırmalar, elektrik enerjisinin insanlık için vazgeçilemez bir enerji çeşidi olduğunu belirtiyor. Şekil 1'de Türkiye'nin yıllık toplam elektrik tüketimi, Şekil 2'de ise kişi başına tüketilen elektrik miktarları gösterilmektedir.

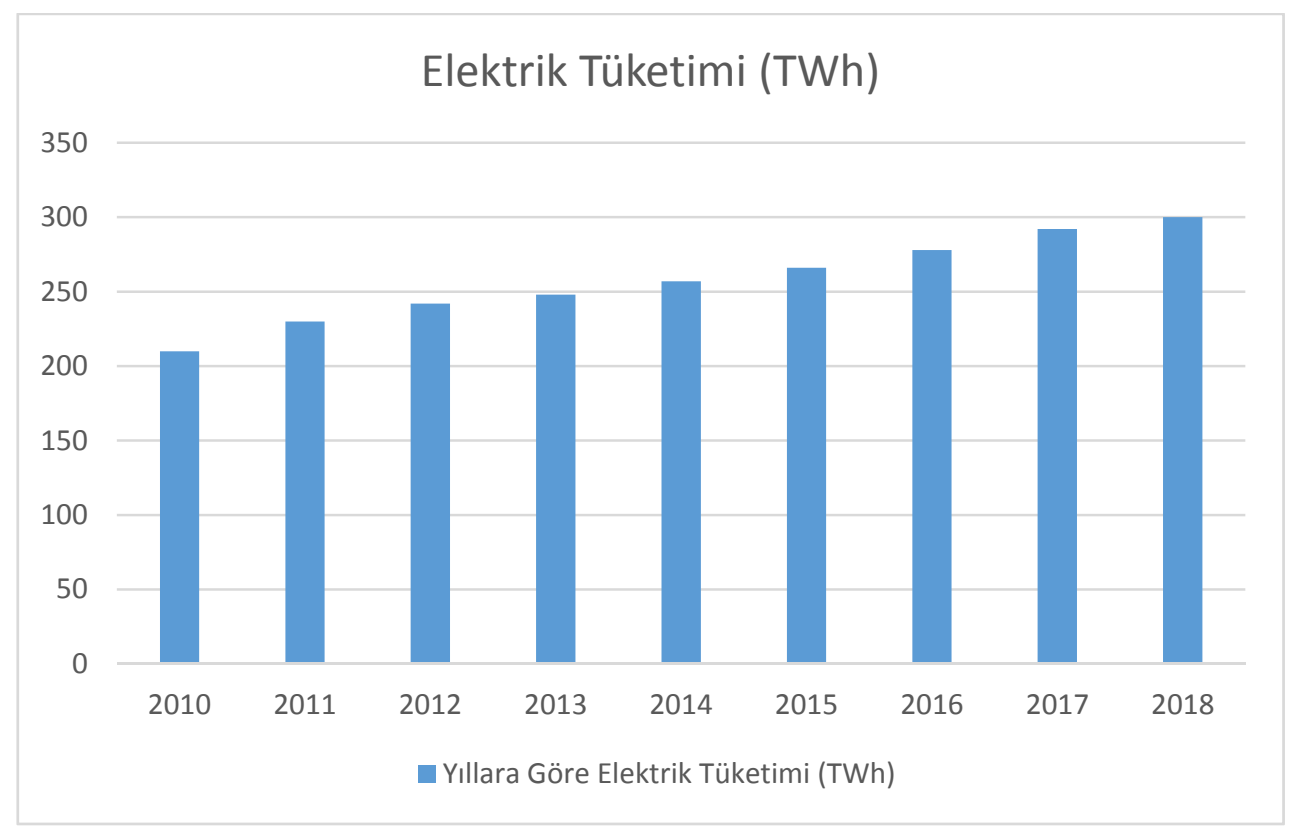

Şekil 1. Türkiye'nin Yıllara Göre Elektrik Tüketimi

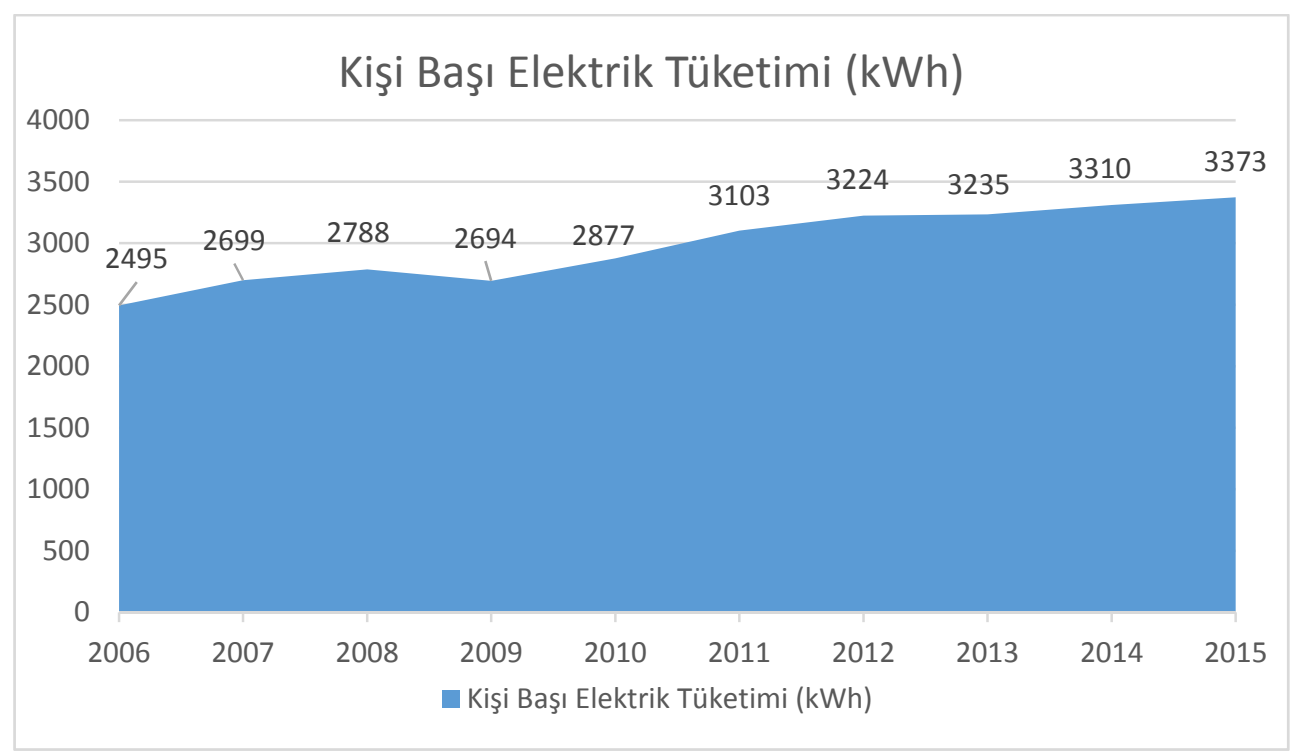

Şekil 2. Türkiye'nin Yıllara Göre Kişi Başı Elektrik Tüketimi 
Dünya üzerinde bulunan enerji kaynakları güneş 1şınımının maddeler üzerindeki fiziksel ve kimyasal etkisinden meydana gelmektedir. Elektrik enerjisi elde etmenin hem ekonomik hem de doğaya zarar vermeden yapılabilmesinin en etkili ve verimli yolu ise sonsuz bir kaynak olan 'Hidrolik Enerji'den yararlanmaktır. Suyun bu enerjisinden faydalanarak elektrik enerjisi üreten sistemlere 'Hidroelektrik Sistemler' adı verilir.

Hidroelektrik sistemlerde suyun kinetik enerjisinden faydalanılır. Bir barajda depolanan su cebri borular vasıtasıyla yüksekten alınıp türbin kısmına verilerek türbin çarkını döndürmeye başlar. Türbin çarkı ile aynı eksende bulunan ve şaft adı verilen miller ile çarka bağlı bulunan jeneratörde aynı dönme hareketini kazanarak elektrik enerjisi üretir. Burada suyun kinetik enerjisinin dönme hareketine çevrilmesini 'Hidrolik Türbinler' sağlar.

Hidrolik Türbinler hava ile temas eden türbinler (Aksiyon Türbinleri) ve hava ile temas etmeyen türbinler (Reaksiyon Türbinler) olarak ikiye ayrılır. Francis Türbinleri hava ile temas etmemekte olup batık halde çalışırlar. Francis türbinleri hem dikey kullanımda hem yatay kullanımda eşit derecede iyi çalıştıklarından orta ve büyük ölçekli hidroelektrik santrallerde en sık kullanılan türbin türüdür.

\section{Materyal ve Metot}

Türbin tasarımında ilk olarak su debisi ve düşüsü bilinen santralin kurulacağı yere en uygun türbin tipi seçimi yapılmalıdır. Daha sonra türbin gücünün belirlenebilmesi için ihtiyaç olan özgül hız- türbin verimi ve türbin devir sayısı gibi parametrelerin belirlenmesi zaruridir. Son olarakta Türbin çarkı ve verime etkilerinden dolayı türbin salyangoz ve türbin emme borusu boyutlandırmaları yapilmalidir.

\subsection{Türbin Tipi Seçimi}

Hidrolik düşü ve su debisi türbin tipi seçiminde belirleyici faktörlerdir. Hidrolik düşüsü ve debisi bilinen sistemin türbin tipi Şekil 3’te gösterilen diyagramdan yararlanarak bulunabilir. 


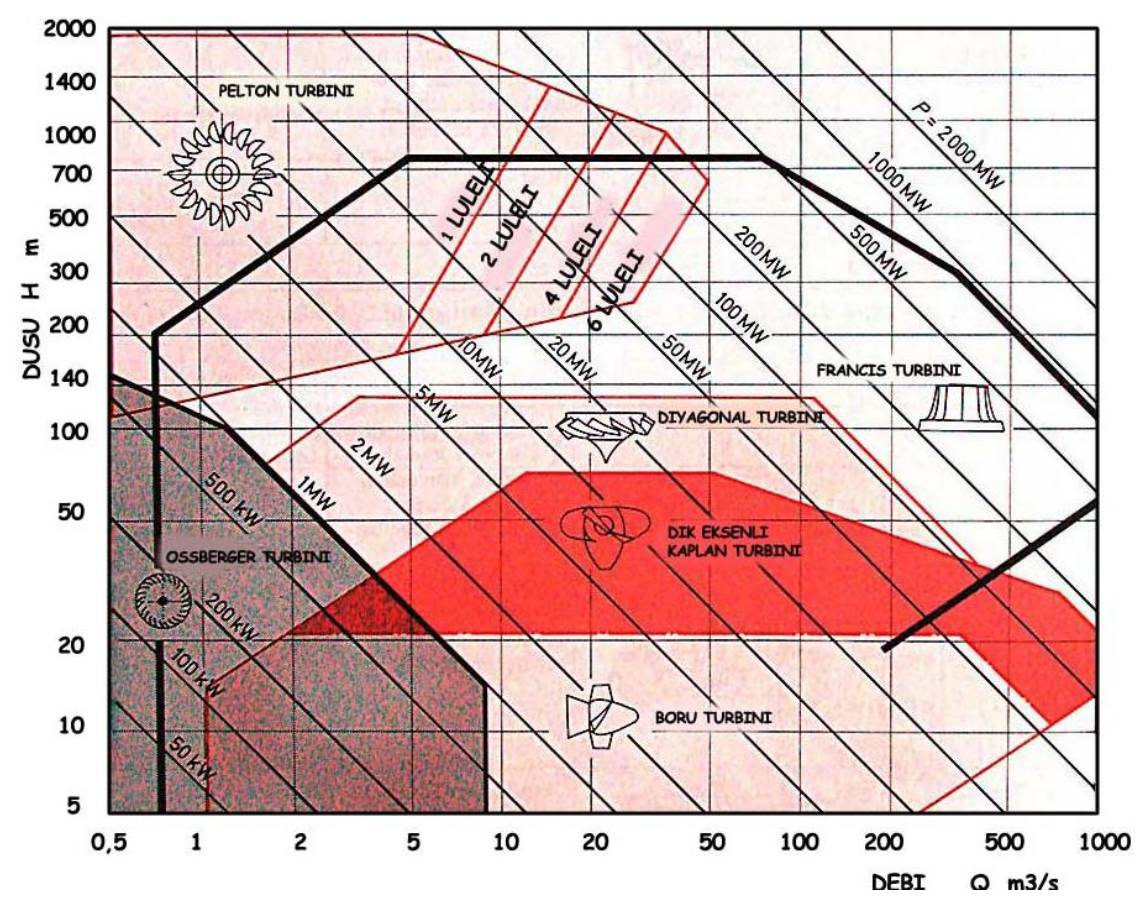

Şekil 3. Düşü ve debi değerlerine göre türbin seçim diyagramı

Türbin tipi seçimi özgül hıza göre de yapılır. Hidrolik türbinlerin özgül devir sayılarının belirlenmesi için değişik imalatçı firmalar ve araştırmacılar bazı ampirik formüller geliştirmişlerdir [20]. Siervo ve Leva'ya göre özgül hız tayini;

$n_{s}=\frac{3470}{\left(H_{d}\right)^{0.65}} \quad,(\mathrm{dev} / \mathrm{dk})$

$H_{d}=$ Net Düşü (m)

olarak hesaplanır. Tablo 1'de Türbin tiplerinin hangi özgül hız aralıklarında çalıştıkları gösterilmektedir.

Tablo 1. Özgül hıza göre türbin tipi seçimi

\begin{tabular}{cc}
\hline Model & Özgül Hız (dev/dk) \\
\hline Pelton & $12-30$ \\
Francis & $80-400$ \\
Kaplan & $340-1000$ \\
\hline
\end{tabular}

\subsection{Türbin Gücü ve Verim}

Hidrolik Türbinlerin güçleri aşağıdaki formüller ile hesaplanır. 
$N=\frac{\gamma \cdot Q \cdot H_{d} \cdot \eta_{t}}{75} \quad, \quad(\mathrm{BG}) \quad$ veya $\quad N=\frac{\gamma \cdot Q \cdot H_{d} \cdot \eta_{t}}{102}, \quad(\mathrm{~kW})$

$\mathrm{N}=$ Türbin gücü $(\mathrm{BG})$ veya $(\mathrm{kW})$,

$\gamma=$ Suyun yoğunluğu $\left(\mathrm{kg} / \mathrm{m}^{3}\right)$,

$\mathrm{Q}=$ Suyun debisi $\left(\mathrm{m}^{3} / \mathrm{s}\right)$,

$H_{d}=$ Dizayn net düşü $(\mathrm{m})$,

$\eta_{t}=$ Türbin verimi

Burada Türbin gücünü etkileyen önemli faktörlerden birisi de Türbin Verimi'dir. Türbin içindeki kayıplar ne kadar az olursa türbin verimi de o kadar yüksek olur. Türbin verimi şu ifadeyle hesaplanır.

$\eta_{t}=\frac{\tau \cdot \omega}{\rho \cdot g \cdot Q \cdot H}$

$$
\begin{aligned}
& \tau=\text { Tork }(\mathrm{N} . \mathrm{m}), \\
& \omega=\operatorname{Açısal~Hız~}(\mathrm{d} / \mathrm{d})=2 \pi \mathrm{N} \\
& \rho=\operatorname{su~yoğunluğu~}\left(\mathrm{kg} / \mathrm{m}^{3}\right), \\
& \mathrm{Q}=\text { Suyun debisi }\left(\mathrm{m}^{3} / \mathrm{s}\right), \\
& \mathrm{g}=\text { Yerçekimi ivmesi }\left(\mathrm{m} / \mathrm{s}^{2}\right)
\end{aligned}
$$

Şekil 4'te hidrolik türbinlere ait karakteristik verim eğrileri gösterilmiştir.

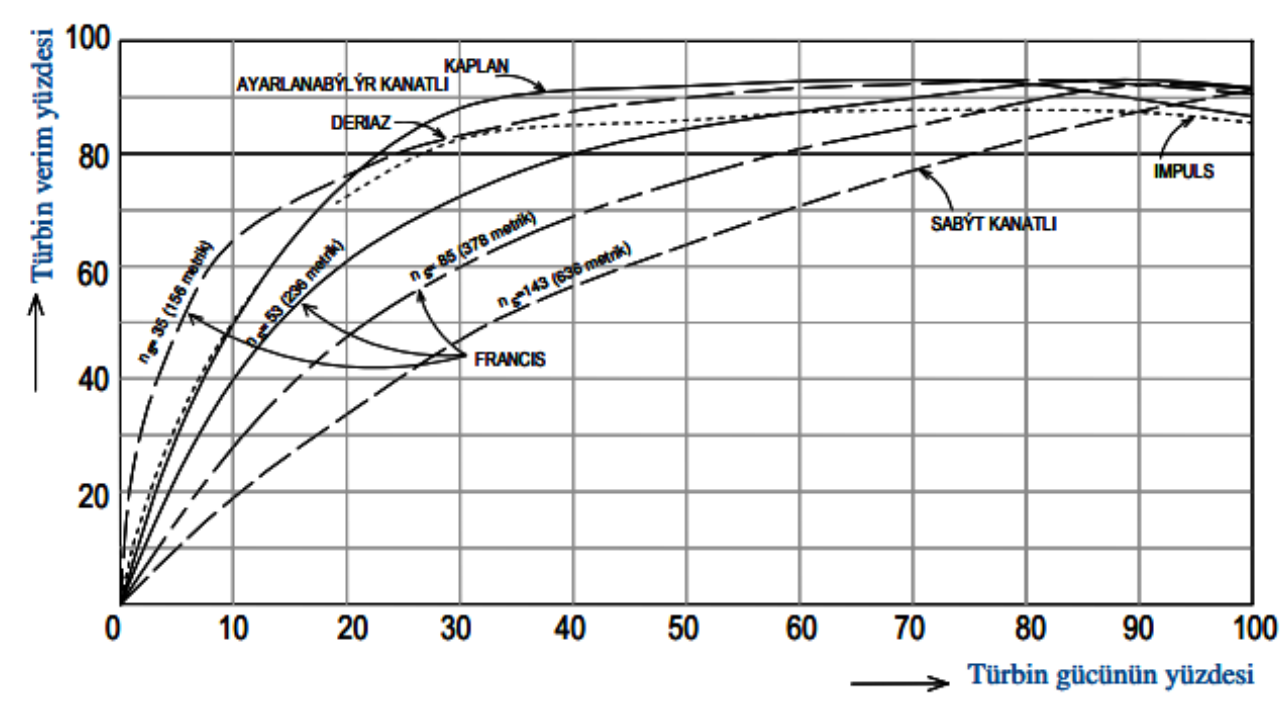

Şekil 4. Hidrolik Türbinlerin Karakteristik Verim Eğrileri 
Türbin verimini etkileyen diğer faktörler kavitasyon ve girdap (Vortex) olayıdır. Kavitasyon oluşmasına neden olan düşük basınçlı hacimcikler akışın dik kesiti daralttığı için gücün düşmesine neden olurlar. Ayrıca vuruntu ve salınımlar türbin verimini $\% 20$ ye kadar düşürebilir. Türbinin kavitasyon katsayısı aşağıdaki formülle hesaplanır.

$$
\sigma_{t}=\frac{H_{a}-H_{v}-H_{s}}{H_{n}}
$$

Burada $H_{a}$ : Atmosfer basıncı (mss), $H_{v}$ : Akışkanın buharlaşma basıncı (mss), $H_{s}$ : Çark ekseni ile çıkış suyu seviyesi yüksekliği (m), $H_{n}$ : Net hidrolik düşü (mss) ifade eder. Türbinin kavitasyonsuz ve en yüksek verimle çalışabilmesi için $\sigma_{t} \geq \sigma_{k r}$ olmalı ve $\sigma_{t}, \sigma_{k r}$ 'ye mümkün olduğunca yakın alınmalıdır.

Kritik kavitasyon katsayısı özgül hıza bağlı olarak;

$\sigma_{k r}=2.464 \times 10^{-7} \cdot n_{s}^{3.01}$

ifade edilir.

Girdap (Vortex) olayı ise jeneratör yüke binmeye başladıktan sonra (takriben \%15-\%20) oluşur, yükün \%45-65 civarında en üst seviye ve yükün \%70-75 seviyesinde ise azalma eğilimi gösterir. Şekil 5 'te ünite yüke bindiği zamanki vukuu bulan girdap olayının davranışı gösterilmiştir 


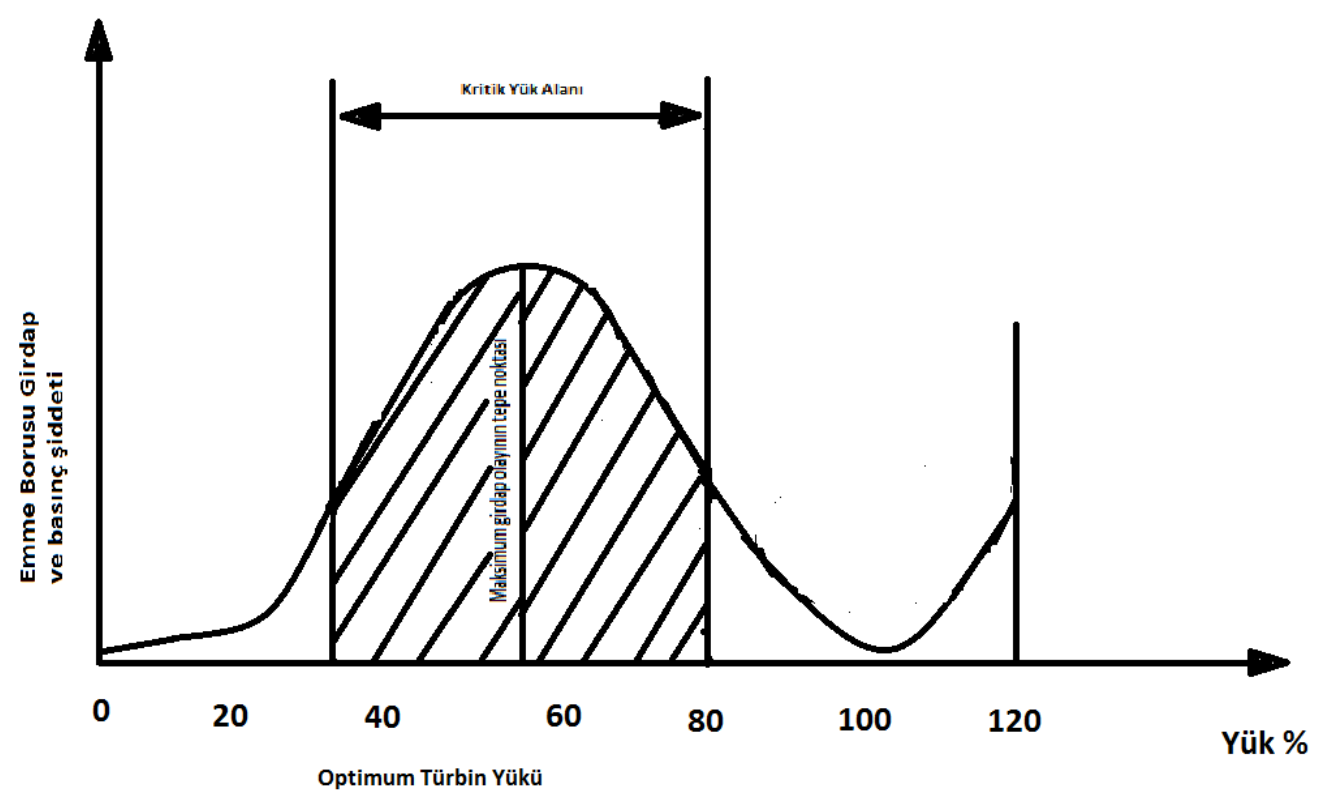

Şekil 5. Girdap olayının optimum yüklerde davranışı

\subsection{Francis Türbin Çarkı Boyutlandırılması}

Francis türbin çarkı tasarımı, düşü ve debi değerlerinin verilmesiyle başlar. Francis türbin çark1, çeşitli firma ve araştırmacıların çalışmaları sonucu türbinin özgül hızına ve rotor çıkış çapına bağlı olarak geliştirilen formüller vasıtasıyla türbin çarkının ana boyutları hesaplanır. Türbin çarkının ana boyutları Şekil 6'da verilmiştir.

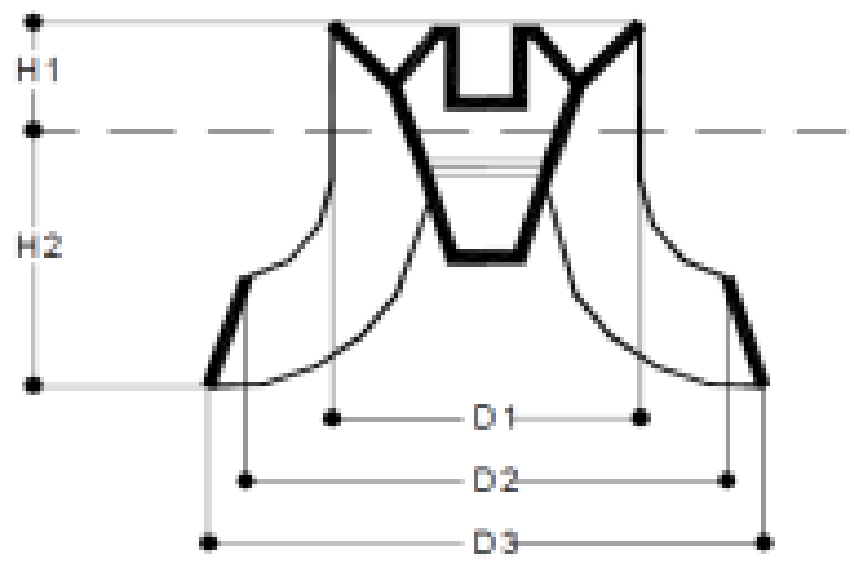

Şekil 6. Francis Türbin Çarkı Boyutları

Francis türbin çarkı ana boyutlarını hesaplamak için önce $D_{3}$ çark çıkış çapının bilinmesi gereklidir. Siervo ve Leva'ya göre Türbin çıkış çapı; 
$D_{3}=84,5 \cdot k_{u} \frac{\sqrt{H_{d}}}{n},(\mathrm{~m})$

şeklinde hesaplanır. Burada $n$; devir sayısı, $H_{d}$; Net düşü, $k_{u}$ ise özgül hıza bağlı katsayıdır ve şu şekilde hesaplanır.

$k_{u}=0,31+2,5 \times 10^{-3} n_{s}$

$D_{3}$ çıkış çapı bulunduktan sonra Türbin çark boyutları bu değere göre hesaplanır. Buna göre $D_{1}$ giriş çap1;

$D_{1}=\left(0,4+\frac{94,5}{n_{s}}\right) \cdot D_{3}, \quad(\mathrm{~m})$

$D_{2}$ çıkış ağzı dar çapı (orta çap);

$D_{2}=\frac{D_{3}}{0,96+0,00038 . n_{s}}, \quad(\mathrm{~m})$

olarak bulunur.

$H_{1}$ yüksekliği;

$H_{1}=\left(0,94+0,000025 \cdot n_{s}\right) \cdot D_{3}, \quad(\mathrm{~m})$

$H_{2}$ yüksekliği ise özgül hıza bağlı olarak;

$$
\begin{array}{ll}
H_{2}=\left(0,05+\frac{42}{n_{s}}\right) \cdot D_{3},(\mathrm{~m}) & 50<\mathrm{n}_{\mathrm{s}}<110 \\
H_{2}=\frac{D_{3}}{3,16-0,0013 \cdot n_{s}},(\mathrm{~m}) & 110<\mathrm{n}_{\mathrm{s}}<350
\end{array}
$$

şeklinde hesaplanır. 
Çark kanat sayısı türbinin gücünü ve kavitasyon karakteristiklerini belirleyen faktörlerden biridir. Kanat sayısının az olması türbin verimini ve özgül hızı yükseltir, yük kaybı azalır. Kanat sayısı fazla olursa da yük kaybı artar. Ayrıca fazla kanat kullanarak suyun geçiş yerleri daraltılırsa aynı debi değeri için hızlar büyür ve kavitasyon tehlikesi artabilir. Yüksek düşülerde kavitasyon tehlikesini azaltmak için kanat sayısı arttırılır. Kanat et kalınlığ 1 ne kadar ince olursa kavitasyon tehlikesi o kadar azalır. Kanat sayısı Tablo 2 yardımıyla bulunur.

Tablo 2. Novalev Özgül Devir Sayısı- Çark Kanat Sayısı İlişkisi

\begin{tabular}{cc}
\hline Özgül devir sayıs1 & Çark Kanat Sayıs1 \\
\hline $60-80$ & $21-19$ \\
$80-120$ & 19 \\
$120-150$ & $19-17$ \\
$150-180$ & 17 \\
$180-200$ & $17-15$ \\
$200-250$ & $15-14$ \\
$250-300$ & 14 \\
$300-350$ & $14-12$ \\
$350-400$ & $12-9$ \\
\hline
\end{tabular}

\section{Bulgular ve Tartışma}

Günümüzde elektrik tüketimi önceki yıllara göre artış göstermiş ve her geçen gün artmaya devam etmektedir. Elektrik enerjisi üretiminde yenilenebilir enerji kaynaklarına yönelmek, sonsuz enerji kaynaklarını kullanmak, dışa bağımlılığın azalması anlamına da gelmektedir. Bu yenilenebilir enerji kaynakları arasında da en verimli olanı hidroelektrik üretim yöntemidir.

$\mathrm{Bu}$ çalışmada Fizibilite çalışması yapılmış Hidroelektrik üretim tesisi kurulacak alanda hangi türbinin kullanılabileceği konusunda düşü ve debi değerlerinden faydalanılarak tablo ve formüller aracılı̆̆ 1 ile türbin tipi belirlenmiştir.

Debi ve düşüye göre hangi türbin tiplerinin kullanılacağı aşağıdaki tabloda verilmiştir.

Tablo 3. Türbin Tipi-Düşü ve Debi İlişkisi

\begin{tabular}{lll}
\hline Türbin Tipi & Düşü & Debi \\
\hline Pelton & Yüksek & Düşük \\
Francis & Yüksek,Orta,Düşük & Düşük,Orta,Yüksek \\
Kaplan & Düşük & Yüksek \\
\hline
\end{tabular}




\section{Sonuçlar ve Öneriler}

Enerji ihtiyacının karşılanmasında yenilenebilir enerji kaynaklarına yönelerek, bu kaynaklar doğrultusunda hamleler yapmak gelecek adına büyük bir öneme arz etmektedir. Yenilenebilir Enerji Kaynaklarına yatırım yapmak hem çevresel hem de hammadde temini bakımından maliyeti en düşük olacağından bu kaynakları harekete geçirmek en doğrusu olacaktır. Diğer üretim tipleri ile karşılaştırıldığında işletme maliyeti, yüksek verim ve uzun işletme ömrü ile Hidroelektrik Santraller ilk sıradadır. Genel anlamda her türlü düşü ve debide kullanılabilen Francis türbini, hidroelektrik santrallerde en yaygın olarak kullanılan türbin çeşididir. Bu çalışmada Francis tipi türbin tasarımında hangi parametrelerin etkili olduğu ifade edilmiştir.

\section{Kaynaklar}

Tekno Tasarım, Hidroelektrik Enerji ve Türbinler, adres @ https://docplayer.biz.tr/5870129Hidroelektrik-enerji-ve-turbinler.html,

Türkiye Elektrik Tüketimi, adres @ https://www.enerjiatlasi.com/elektrik-tuketimi//, Erişim Tarihi: 09.02.2019

Dang, P., Hanania, J., Stenhouse, K., Donev, J., Francis Turbine, adres @ https://energyeducation.ca/encyclopedia/Francis turbine

Köse, F., Türbin Karakteristik Deneyleri ile Kanat Verimliliğinin Iyileştirilmesi Iç̧in Bir Francis Türbini Deney Setinin Kurulması ve Araştırılması, Doktora Tezi, Konya, 1996

Erdem, M., Küçük Hidroelektrik Santrallerin Tasarım Ölçütleri, Yüksek Lisans Tezi, Denizli, 2006

Hidrolektrik Enerji, adres@ @ http://eng.harran.edu.tr/ ccetiner/hidro_elektrik_enerji_2.pdf Erişim Tarihi: 03.05.2019

T.C. Tarım ve Orman Bakanlığı, Devlet Su İşleri Genel Müdürlüğü, 2018 Faaliyet Raporu, adres@ http://www.dsi.gov.tr/docs/stratejik-plan/dsi-2018-faaliyet-raporu.pdf?sfvrsn=2 Erişim Tarihi: 09.02.2019

Başeşme, H., Hidroelektrik Santraller ve Hidroelektrik Santral Tesisleri, EÜAŞ Yayınları, Ankara (2003)

Bulut, M., Hidroelektrik Enerji ve Hidroelektrik Santrallerde Türbin Tipi Seçiminin Verime Etkisinin Incelenmesi, Yüksek Lisans Tezi, Gümüşhane, 2013 